\title{
Simultaneous in situ Detection of the Optical Fluorescence, Fluorescence Recovery Kinetics After Photobleaching \& Membrane Ion Flux on the Electrophysiological Lab-on-a-Chip
}

\author{
Paul Alexandrov ${ }^{1}$, Alexander Notchenko ${ }^{2}$, Margaret Gradova ${ }^{3}$, Oleg Gradov ${ }^{1}$, * \\ ${ }^{1}$ Talrose Institute for Energy Problems of Chemical Physics, RAS, Moscow, Russia \\ ${ }^{2}$ Institute for Information Transmission Problems, RAS, Kharkevich Institute, Moscow, Russia \\ ${ }^{3}$ Semenov Institute of Chemical Physics, RAS, Moscow, Russia
}

Email address:

gradov@center.chph.ras.ru (O. Gradov)

\section{To cite this article:}

Paul Alexandrov, Alexander Notchenko, Margaret Gradova, Oleg Gradov. Simultaneous in situ Detection of the Optical Fluorescence, Fluorescence Recovery Kinetics After Photobleaching \& Membrane Ion Flux on the Electrophysiological Lab-on-a-Chip. American Journal of Optics and Photonics. Vol. 3, No. 5, 2015, pp. 118-122. doi: 10.11648/j.ajop.20150305.19

\begin{abstract}
The need for simultaneous data recording from multiple channels and synchronization of the correlation data processing in patch-clamp on microelectrode arrays / chips with many data capture points corresponding to single channel ionic kinetic processes of individual cells leads to the idea that adaptive variation of the local potential registration conditions in multichannel devices without signal preprocessing in real time is impossible. Moreover, the advisability of direct registration coupling with the model realization for kinetic identification of the process during patch-clamp can be realized only in case of their synchronization. We propose here such a measurement system.
\end{abstract}

Keywords: FRAP, Fluorescence Recovery Kinetics, Lab-on-a-Chip, Photobleaching, Photovoltage Clamp, Real Time Target Machine, Patch-Clamp, Ion Flux

\section{Introduction}

One of the most powerful trends in the local membrane potential fixation technique, known as patch clamp, is the channel virtualization and synchronization of a real experiment with a virtual simulation of the process on a PC. The early techniques of the 1980-th allowed only the grounding virtualization or computer-aided level stabilization [1], but in the 2000-th a new trend appears for combining dynamic registration technique (so called dynamic clamp) of single ion channels with their real-time simulation [2-4].

The adequate ion channel models under various treatments, obtained during synchronous electrophysiological and computational experiments allow the development of "virtual reality" for clinical neurophysiology, in which it will become possible to simulate the state of excitable cell membrane structures under various stimuli [5], and thus, to vary in the course of a real-time experiment the affecting parameters in order to predict the most likely response of the system, according to the preliminary simulation data. In some cases virtual screening together with the automated patch-clamp registration is applied for studying selective ion channel inhibitors [6] and other membrane-bound agents, which can not be discussed here in details for the reason of a limited format of this paper.

However, mass patch-clamp techniques, such as planar patch clamp $[7,8]$ or population patch clamp $[9,10]$ using labs-on-a-chip and other microfluidic devices [11] are usually applied for the purposes of screening and representative analysis of cell populations. At the same time the synchronous multi-channel monitoring complexation with a real-time analysis during registration from many cells as signal sources (i.e. multiple ion channel registration) was problematic, therefore the design of devices for patch-clamp registration with synchronous data processing and computational simulation for this additive method has not actually been described in literature.

The known pharmacological approaches for screening during patch-clamp (see $[12,13]$ ) are almost qualitative and can only answer the biomedical questions but fail to provide 
signal form approximation with automatic fitting in real time. This results in the fact that the approaches for virtualization of neurophysiological patch-clamp control degrade from the creation of virtual reality with a cell-machine interface to single virtual simulation [14], which differs strongly in the computational complexity, but not in the purpose from the known computer simulation methods used in 1970-th - 1980th. $[15,16]$.

In this connection there is a strong need in designing of special devices for data analysis in patch-clamp experiments together with the real time computer simulation. The feasibility of hard and soft real-time regimes differ with the type of platforms and operating systems, so it is necessary to develop and implement the optimized patch-clamp configuration, capable of simultaneous registration, calculation / simulation, virtualization and comparative processing of the sourced data in real time using the most appropriate hardware.

\section{Technology: Data Acquisition}

Using MATLAB / SIMULINK we provided the signal recording to the sound card via a specially designed converter. The ion channel models used for comparison with the really obtained kinetic data were to operate in real time. It was shown that with the input unit (from the audio input) the model operates in real time; in alternative registration schemes it was also possible to achieve a real-time mode in Windows by intercepting the interrupts from the processor. However, a sound card possesses a limited number of channels, which contradicts the idea of multi-channel data registration in the patch-clamp methods from arrays, and thus a hardware realization of multi-channel data acquisition and processing in real time irrespective from a sound card or an audio-input hardware emulation is required.

There is a known «SIMULINK REAL-TIME» technology, based on computers like "xPC TargetBox» connected to the Windows-terminal operating as a host machine with a preloaded configuration MATLAB + SIMULINK $(+\mathrm{xPC}$ Target) for actuator control and post-processing of data obtained from the sensors with the interface commutation through analog-to-digital converting units to the real time target machine / targetbox with the latter provided with a real time operating system (RTOS) like Free DOS and xPC Target kernel, a kernel-level package transfer utility and a driver package xPC Target. As a consequence the above configuration allows to obtain maximum information from the experimental runs with the real-time data processing.

We propose to apply this approach for the implementation of the multi-channel patch-clamp. For this purpose the registration channels should be commutated through the conversion units to the real-time machine interfaces. The technical implementation of this principle is not a significant problem, but varies depending on the target real time computer. Thus the "Modular" model in its standard version has 7 slots for input-output modules, in a minimal (small) modification - 6 and in the extended (large) one - 13. The modification type depends on the required number of inputoutput modules determined by the nomenclature of labs-on-achip / electrode matrix, and, consequently, by the studied samples. Furthermore, the connection of the general-purpose input-output modules is possible through RS232 / 422 / 485 (up to $115 \mathrm{kbit} / \mathrm{c}$ ), commonly used for interfacing the host machine and the target computer. We have also tested a compact "Mobile" model with SPP / EP / ECP - IEEE1284 parallel port as a real time machine, but it is somewhat inferior in performance to the stationary modification, and thus can not be recommended for massively work.

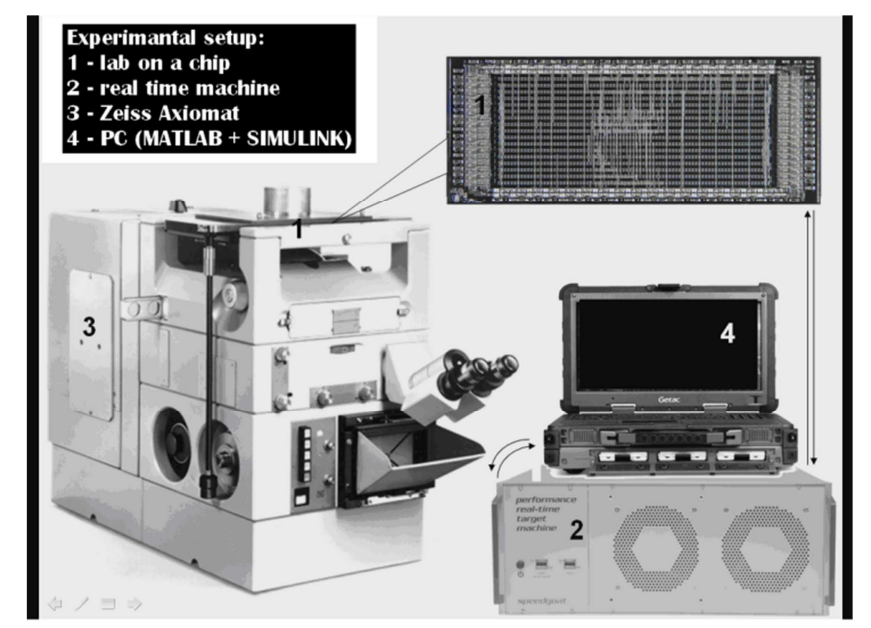

Fig. 1. The experimental setup scheme (without ADC and other imbedded supplementary appliances). From our presentation for MSW-2014 and RGC2014.

In order to facilitate the user interaction with the system for biologists without special computer skills in MATLAB or SIMULINK and those who prefer not to deal in the real time technologies, a special software shell was designed - an autonomous graphical user interface with the GUI in a MATLAB-independent window. Despite the fact that as a result of the above procedure the total size of the software increases by some hundreds of megabytes as compared with the code file size (due to the necessity to transfer the corresponding libraries to the PC without preinstalled Math Works), the control ease for the operator unfamiliar with high-level programming fully covers this drawback.

\section{Technology: Optics and Fluorescence Kinetics}

Since during the microscope-assisted patch clamp it is crucial to know which cells are the source of the signal observed, one can apply fluorescent labels with the luminescence efficiency being proportional to the ion channel activity. We have developed a novel system for pattern recognition of the labeled cells during the real time patch clamp controlled by the single-board computer "Raspberry $\mathrm{Pi}^{\prime}$ with the real time operating system Windows CE based on Win32 API using the dynamic link libraries. Later we attempted to implement porting on / to Arduino using the real 
time clock/timer DS1307 applying the $12 \mathrm{C}$ protocol with the $1 \mathrm{~Hz}$ output channel. However, this design was not implemented into the laboratory practice due to its low performance. In general, the fluorescent control over the biological process for the user included an automatic identification of the luminescent cells with the geometric figures (markers).

Knowing the correspondence between the marker location on the microscopic image and the distribution of the sensitive elements in the patch clamp CCD / CMOS chip (sensels), it is possible to select a particular ion channel for morpho- $/$ electrophysiological analysis based on the automatically detected luminescent regions.

Moreover, the above system can be applied to the FRAP (Fluorescence Recovery After Photobleaching) measurements on a chip, where the shape of the luminescent area should be morphologically controlled in order to perform a number of diffusion-coupled parameters. The combination of the membrane diffusion FRAP-based measurements with the determination of the ion currents and transmembrane transport parameters using patch clamp technique performed on a fluidic chip is an innovative technology which allows not only the determination of the native diffusion and transport parameters, but also the real time registration of the chip response to the filling with the different chemical agents (capable of either interaction with the membrane or penetration through it).

Moreover, the software designed by our coauthor Notchenko A.V. (Institute for Information Transmission Problems RAS) it is possible to visualize the vector fields with their form and direction indicating the diffusion process activity. This option becomes available after putting a tick at the «Show Flow» section of the user menu (see. Fig. 2).

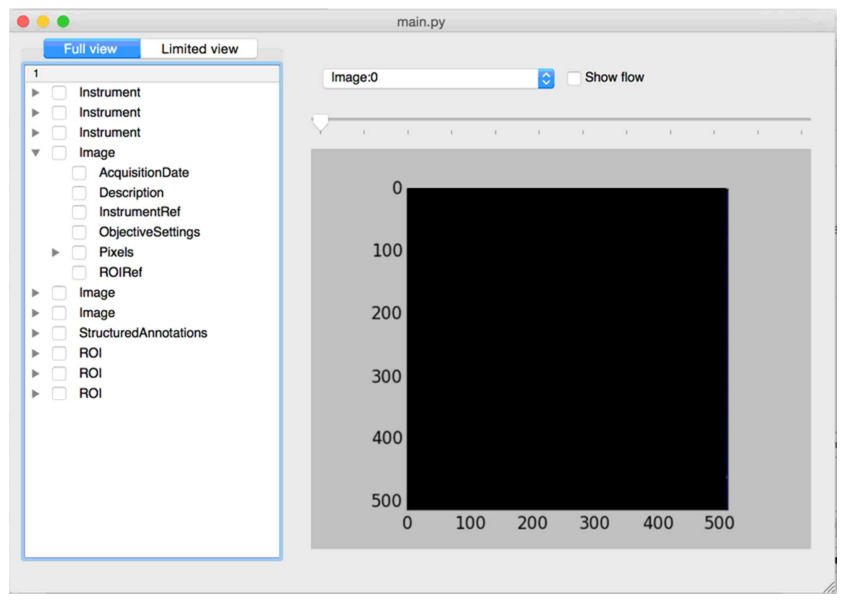

Fig. 2. Vector field FRAP / FLIP analyzer for diffusion measurements.

The advantages of the above approach include the userfriendly interface and the presence of the feedback which allows to perform the automatic control over the setup parameters depending on the state of the fluorescent and patch-clamp active cells. As a consequence, it is possible to provide a double system verification not only by means of the model fitting running with a several real-time machine, but also using a fluorescent signal from the cells, resulting in a cross-control in the patch clamp on-a-chip.

Since many contemporary works describe a combination of the patch clamp technique with the optical measurements $[17,18]$ (with the fluorescent method for the target search of the local potential fixation coordinates using a micropipette being the most advanced method [19]), the application of the real-time operation regime to the patch clamp with the parallel optical detection seems to be a very useful improvement, because the existing problem of designing the "optical patch clamp" [20] with the synchronized optical detection and electrophysiological measurements with the virtualization can be solved only using the real-time machines.

The Raspberry Pi-based design of the setup allows to construct a data processing cluster with a number of singleboard computers of the same class, capable of simultaneous data processing from several labs-on-a-chip with the subsequent data transmission via the buses to the terminal device. It is noteworthy that the presence of Ethernet and two universal serial data interfaces (USB 2.0) in the B-version of the above system allows the bidirectional data transmission at the time intervals optimal for the cellular measurements, including the longitude monitoring at the remote access.

The system described was assembled in a single package, except from the external camera. For the purpose of distribution of the computational data flows from multiple labs-on-a-chip we assembled a cluster rack (crate) with several servers used as the host machines, as well as the systems of the advanced mathematical data processing (not in the real-time mode). It should be noted that it is impossible to construct an equivalent system using the single-board computers without the above-mentioned technical solutions, since both Raspberry Pi and Arduino lack the built-in real time clock (RTC) at the board [21]. The application of modular standardization in design of such DIY-setups allows to differentiate them according to their destination without using multiple duplicated processing units, which is a significant benefit for construction of the flexible real-time experimental hardware \& software systems.

Thus, we designed a complex measurement system which, unlike the previously known systems for real-time patch-clamp on-a-chip, allows not only registration and screening [22] or a simultaneous optical detection and electrophysiological measurements [23], but also a comparison with the SIMULINK model with both the measurement and simulation data transmission to either a host machine or to the local network through the Ethernet protocol.

\section{Perspectives}

The above concept gave rise to the experiments on the patch clamp of the synthetic ionic channels and artificial cells, as well as to the hybridization of the real-time patch clamp with the mass spectrometric monitoring (the so-called MS-patch-clamp) [24], which are the continuation of the work initiated by the authors in the early 2000-th. 
In recent years, the prospects of combination of the MS MALDI-imaging with FRAP [25, 26] suggest the feasibility of the MALDI-FRAP-measurements on chips performed simultaneously with the MS-patch-clamp. Currently, there is a growing number of studies on the MALDI-on-chip, and the hybridization of the above technologies is expected to provide a vast amount of new experimental data for cytology, electrophysiology, molecular biology and biophysics.

\section{Acknowledgments}

The authors gratefully acknowledge the contribution of the PhD students Zaytsev E. V. (D.Mendeleyev University of Chemical Technology of Russia) and their foreign colleagues for the fruitful collaboration.

\section{References}

[1] J. Lopez-Barneo, D. R. Matteson and C. M. Armstrong, "Currents recorded through small areas of squid axon membrane with an internal virtual ground voltage clamp", Biophys. Journ., vol. 36, no. 3, pp. 811-815, 1981.

[2] A. D. Dorval, D. J. Christini and J. A. White, "Real-Time linux dynamic clamp: a fast and flexible way to construct virtual ion channels in living cells", Ann. Biomed. Eng., vol. 29 , no. 10 , pp. 897-907, 2001.

[3] D. W. Wheeler, P. H. Kullmann and J. P. Horn, "Estimating use-dependent synaptic gain in autonomic ganglia by computational simulation and dynamic-clamp analysis", Journ. Neurophysiol., vol. 92, no. 5, pp. 2659-2671, 2004.

[4] J. C. Bettencourt, K. P. Lillis, L. R. Stupin and J. A. White, "Effects of imperfect dynamic clamp: computational and experimental results", Journ. Neurosci. Meth., vol. 169, no. 2, pp. 282-289, 2008.

[5] M. N. Economo, F. R. Fernandez and J. A. White, "Dynamic clamp: alteration of response properties and creation of virtual realities in neurophysiology", Journ. Neurosci., vol. 30, no. 7, pp. 2407-2413, 2010.

[6] S. J. Wacker, W. Jurkowski, K. J. Simmons, C. W. Fishwick, A. P. Johnson, D. Madge, E. Lindahl, J. F. Rolland and B. L. de Groot, "Identification of selective inhibitors of the potassium channel $\mathrm{Kv} 1.1-1.2_{(3)}$ by high-throughput virtual screening and automated patch clamp", Chem. Med. Chem., vol. 7, no. 10, pp. 1775-1783, 2012.

[7] E. S. Trepakova, M. G. Malik, J. P. Imredy, J. R. Penniman, S. J. Dech and J. J. Salata, "Application of PatchXpress planar patch clamp technology to the screening of new drug candidates for cardiac KCNQ1/KCNE1 $\left(I_{\mathrm{Ks}}\right)$ activity", Ass. Drug Dev. Techn., vol. 5, no. 5, pp. 617-627, 2007.

[8] C. Farre and N. Fertig, "HTS techniques for patch clampbased ion channel screening - advances and economy", Exp. Opin. Drug Discov., vol. 7, no. 6,. pp. 515-524, 2012.

[9] E. C. Hollands, T. J. Dale, A. W. Baxter, H. J. Meadows, A. J. Powell, J. J. Clare and D. J. Trezise, "Population patch-clamp electrophysiology analysis of recombinant $\mathrm{GABA}_{\mathrm{A}} \alpha 1 \beta 3 \gamma 2$ channels expressed in HEK-293 cells", Journ. Biomol .Screen., vol. 14, no. 7, pp. 769-780, 2009.
[10] V. H. John, T. J. Dale, E. C. Hollands, M. X. Chen, L. Partington, D. L. Downie, H. J. Meadows and D. J. Trezise, "Novel 384-well population patch clamp electrophysiology assays for $\mathrm{Ca}^{2+}$-activated $\mathrm{K}^{+}$channels", Journ. Biomol. Screen., vol. 12, no. 1, pp. 50-60, 2007.

[11] J. Xu, A. Guia, D. Rothwarf, M. Huang, K. Sithiphong, J. Ouang, G. Tao, X. Wang and L. Wu, "A benchmark study with sealchip planar patch-clamp technology", Ass. Drug Dev. Technol., vol. 1, no. 5, pp. 675-684, 2003.

[12] R. L. Schrøder, S. Friis, M. Sunesen, C. Mathes and N. J. Willumsen, "Automated patch-clamp technique: increased throughput in functional characterization and in pharmacological screening of small-conductance $\mathrm{Ca}^{2+}$ releaseactivated $\mathrm{Ca}^{2+}$ channels", Journ. Biomol. Screen., vol. 13, no. 7, pp. 638-647, 2008.

[13] M. Asmild, N. Oswald, K. M. Krzywkowski, S. Friis, R. B. Jacobsen, D. Reuter, R. Taboryski, J. Kutchinsky, R. K. Vestergaard, R. L. Schrøder, C. B. Sørensen, M. Bech, M. P. Korsgaard and N. J. Willumsen, "Upscaling and automation of electrophysiology: toward high throughput screening in ion channel drug discovery", Recept. Channels, vol. 9, no. 1, pp. 49-58, 2003.

[14] A. Tchaptchet, H. Schneider and Y. F. Braun, "Virtual neurophysiology laboratories for life science education: action potentials and voltage-/patch-clamp recordings", BMC Neurosci., vol. 14, suppl. 1, p. 381, 2013.

[15] J. W. Moore, F. Ramón and R. W. Joyner, "Axon voltageclamp simulations. I. Methods and tests", Biophys. Journ., vol. 15, no. 1, pp. 11-24, 1975.

[16] T. R. Chay, "Kinetic modeling for the channel gating process from single channel patch clamp data", Journ. Theor. Biol., vol. 132, no. 4, pp. 449-468, 1988.

[17] G. J. Augustine, "Combining patch-clamp and optical methods in brain slices", Journ. Neurosci Meth., vol. 54, no. 2, pp. 163169, 1994.

[18] M. K. Park, A. V. Tepikin and O. H. Petersen, "What can we learn about cell signalling by combining optical imaging and patch clamp techniques?", Pflugers Arch.: Eur. Journ. Physiol., vol. 444, no. 3, pp. 305-316, 2002.

[19] D. Ishikawa, N. Takahashi, T. Sasaki, A. Usami, N. Matsuki and Y. Ikegaya, "Fluorescent pipettes for optically targeted patch-clamp recordings", Neur. Net. vol. 23, no. 6, pp. 669672,2010 .

[20] A. Demuro and I. Parker, "Optical patch-clamping": singlechannel recording by imaging $\mathrm{Ca}^{2+}$ flux through individual muscle acetylcholine receptor channels", Journ. Gen. Physiol., vol. 126, no. 3, pp. 179-192, 2005.

[21] S. Bell, Beginning Sensor Networks with Arduino and Raspberry Pi. New York: Springer Sci. + Bus. Media - Apress Media (California), 2013, 345 p.

[22] P. Pathak, H. Zhao, Z. Gong, F. Nie, T. Zhang, K. Cui, Z. Wang, S. T. Wong and L. Que, "Real-time monitoring of cell viability using direct electrical measurement with a patchclamp microchip", Biomed. Microdevices., vol. 13, no. 5, pp. 949-953, 2011.

[23] R. Blunck, D. M. Starace, A. M. Correa and F. Bezanilla, "Detecting rearrangements of shaker and $\mathrm{NaChBac}$ in real-time with fluorescence spectroscopy in patch-clamped mammalian cells", Biophys. Journ., vol. 86, no. 6, pp. 3966-3980, 2004. 
[24] O. Gradov and M. Gradova, "On the possibility of "MS-patchclamp" or mass spectrometry hybridization with patch-clamp setups for single cell metabolomics and channelomics", In: Structure \& Function of Biomembranes (International Workshop "Biomembranes'14"), MIPT, 29 September 2014 3 October 2014, p. 105.

[25] A. Jablokow and O. Gradow, "Verifying Continuity of Membranous Organelles and Measurements of Exchange Rate Between the Nucleus and Cytoplasm using FLIP-Like MALDI-Based Imaging", 63-rd ASMS Conf. on Mass Spectr.
\& Allied Topics (2015) DOI: 10.13140/RG.2.1.2322.3203 [see also: JASMS, Vol. 26, Suppl. 1, p. 169 - ThP 662; DOI: $10.1007 / s 13361-015-1158-2]$

[26] A. Jablokow and O. Gradow, "MS-FRAP or MALDI Imaging Setups With Programmable Laser Sources: a New Way to the Diffusion, Molecular Mobility and Binding Measurements", 63-rd ASMS Conf. on Mass Spectr. \& Allied Topics (2015) DOI: 10.13140/RG.2.1.4919.1841 [see also: JASMS, Vol. 26, Suppl. 1, p. 63 - MP 175; DOI: 10.1007/s13361-015-1158-2]. 